\title{
Universality class of little string theories
}

\author{
Anton Kapustin* \\ School of Natural Sciences, Institute for Advanced Study, Olden Lane, Princeton, New Jersey 08540
}

(Received 28 April 2000; published 28 March 2001)

\begin{abstract}
We propose that little string theories in six dimensions are quasilocal quantum field theories. Such field theories obey a modification of Wightman axioms which allows Wightman functions (i.e. vacuum expectation values of products of fundamental fields) to grow exponentially in momentum space. Wightman functions of quasilocal fields in $x$-space violate microlocality at short distances. With additional assumptions about the ultraviolet behavior of quasilocal fields, one can define approximately local observables associated with big enough compact regions. The minimum size of such a region can be interpreted as the minimum distance which observables can probe. We argue that for little string theories this distance is of the order of $\sqrt{N} / M_{s}$.

DOI: 10.1103/PhysRevD.63.086005

PACS number(s): $11.25 . \mathrm{Mj}$
\end{abstract}

\section{INTRODUCTION}

Poincaré-invariant theories in six dimensions have been much studied in the past few years. One of the reasons is that they describe $(5+1)$-dimensional branes which play an important role is string dualities. In particular, the superconformal $(2,0)$ theory describing several coincident M5-branes has attracted a lot of attention. Another reason is that the very existence of consistent nontrivial Poincaré-invariant theories in six dimensions came as a surprise.

A standard strategy to construct a nontrivial field theory is to take a free conformal theory and perturb it by a relevant or marginally relevant operator other than the mass term. This method works well in dimension four or lower, but it is easy to see that in higher dimensions free conformal theories do not have interesting relevant or marginally relevant deformations. Hence until a few years ago it was believed that nontrivial quantum field theories do not exist in dimensions higher than four.

$(2,0)$ and $(1,0)$ superconformal field theories (SCFTs) describing various fivebranes provided first examples to the contrary [1-5]. Subsequently other related SCFTs in six dimensions have been discovered [6,7]. A common feature of all these constructions is that they require taking a certain limit in string theory or $\mathrm{M}$ theory in which gravity and other bulk modes decouple from the fivebranes. In the simplest case, one takes $N$ coincident type IIA fivebranes or $E_{8} \times E_{8}$ heterotic fivebranes and considers the limit $g_{s} \rightarrow 0, M_{s}$ $\rightarrow \infty$. Here $g_{s}$ is the string coupling at spatial infinity, and $M_{s}=1 / \sqrt{\alpha^{\prime}}$ is the string scale. If gravity and the rest of the bulk modes decouple, while the degrees of freedom living on the brane remain interacting, one expects that the brane degrees of freedom are described in this limit by a nontrivial Poincaré-invariant theory. In all known cases the argument for decoupling is indirect, and the structure of the Poincareinvariant theory is poorly understood.

Nevertheless, whenever one can argue decoupling in the limit $g_{s} \rightarrow 0, M_{s} \rightarrow \infty$, it is believed that the Poincaré-invariant theory is a local quantum field theory (QFT); moreover, it is

*Email address: kapustin@ias.edu a (super)conformal QFT, since $M_{s}$, the only scale in string theory, is taken to infinity.

For reasons mentioned above, these superconformal QFTs cannot be obtained by perturbing a free field theory with a local operator and therefore are not associated to any Lagrangian. But there are nonlocal theories in $5+1$ dimensions which flow to our SCFTs in the infrared: the so-called little string theories (LSTs). Little string theories first appeared in [8] where it was suggested that they describe M theory compactified on $T^{5}$. The precise definition of LSTs as decoupled theories on five branes goes as follows [9]. One starts with $N$ coincident type IIA or heterotic five branes, as above, but now takes the limit $g_{s} \rightarrow 0$ while keeping $M_{s}$ finite. Suppose this limit defines a Poincaré-invariant theory in $5+1$ dimensions. Its infrared limit is equivalent to the limit $M_{s} \rightarrow \infty$, therefore by definition this theory flows to the SCFT of interest. The difficult part is arguing that the decoupling really occurs.

All known nontrivial $(2,0)$ and $(1,0)$ superconformal theories in six dimension arise as the infrared limit of little string theories. In each case the parent little string theory has the same amount of supersymmetry, but does not have conformal or superconformal symmetry. Starting from a slightly different brane configuration, one can also construct little string theories in six dimensions with $(1,1)$ supersymmetry [9]. According to Nahm's classification of superconformal algebras [10], such a theory cannot flow to a nontrivial superconformal theory in the infrared. Instead, at low energies $(1,1)$ theories reduce to $N=2 d=6$ super Yang-Mills theories which are infrared-free.

The name "little string theory" has the following origin. Since we did not send $M_{s}$ to infinity when taking the decoupling limit, it is natural to expect that the theory retains some stringy features. And indeed, one can argue that little string theories inherit from string theory such properties as $T$ duality [9] and Hagedorn density of states [11].

The property of $T$ duality in particular seems to imply that little string theories are not local quantum field theories (despite being Poincaré-invariant). Intuition tells us that a quantum field theory always "knows" on which pseudo Riemannian manifold it lives, while $T$ duality means that a little string theory on a torus of volume $V$ is indistinguishable from a little string theory on a torus of volume $1 / V$. (We 
make this argument more precise in Sec. II.)

Since a common lore says that the local quantum field theory framework is the only way to reconcile quantum mechanics, Poincaré invariance, and macrocausality (see e.g. [12]), we seem to be facing a puzzle. As we explain below, the lore is incorrect if one is willing to sacrifice the notion of a strictly local observable. We will see that there is a way to modify the axioms of quantum field theory so that it is impossible to construct observables whose support is a compact set. In these theories local observables emerge only at distances much larger than a certain scale $l$.

Nonlocal quantum field theories of this sort will be called quasilocal, since they violate causality only at distances shorter than $l$. We conjecture that LSTs are quasilocal quantum field theories with $l$ being of order $\sqrt{N} / M_{s}$. Here $N$ is the number of five branes.

Quasilocal field theory is quite an old subject (see [13] for a review). It was extensively studied in 1960s and 1970s in attempts to deal with nonrenormalizable field theories. The main observation of the present paper is that the known properties of LSTs seem to fit perfectly into the framework of quasilocal field theory.

The link between quasilocal field theories and LSTs is best seen from the holographic point of view [14,15]. Holographic approach makes it clear that little string theories have properties very similar to those of local QFTs. In particular, they have operators $\phi(p)$ which depend on the 6-momentum $p$ whose correlators (= Wightman functions) enjoy most of the usual properties. The only peculiarity of LSTs is that these correlators seem to grow exponentially in momentum space $[15,16]$ (in local QFTs Wightman functions grow at most as a power). A related fact is the exponential growth of the density of states in LSTs [11]. The importance of the exponential growth has been stressed by Aharony and Banks [11]. These authors pointed out that exponential growth means that truly local observables in LSTs do not exist. Rather, if the Wightman functions grow as $\exp (l p)$, the minimal size the observables can probe is of order $l$. Aharony and Banks suggested that the exponential growth of Wightman functions in momentum space is the defining property of LSTs.

In this paper we elaborate on the point made in [11] and clarify the extent to which observables in LSTs can be localized. We will see that the exponential growth of Green's functions in momentum space is characteristic of a quasilocal field theory. Its $x$-space counterpart is the fact that quasilocal fields are very singular (but well-defined) distributions. The corresponding test functions are real-analytic and cannot have compact support.

To appreciate the relation between the growth of distributions in momentum space and the properties of test functions in $x$-space, the following one-dimensional example is helpful. Suppose we want to regard the functions $\widetilde{F}_{ \pm}(p)=e^{ \pm a p}$ of one real variable $p$ as distributions on a suitable space of test functions. (Here $a$ is a positive real number.) It is clear that the space of test functions must include only functions which decay faster than $e^{-|a| p}$ at infinity. For example, one could take the space of smooth functions which are bounded by a multiple of $e^{-(|a|+\delta) p}$ for some positive $\delta$. What is the $x$-space analogue of this condition? The formal Fourier transform of $\widetilde{F}_{ \pm}(p)$ is

$$
F_{ \pm}(x)=\sum_{n=0}^{\infty} \frac{( \pm i a)^{n}}{n !} \delta^{(n)}(x) .
$$

Formally, the value of this distribution on a test function $f(x)$ is

$$
F_{ \pm}(f)=\sum_{n=0}^{\infty} \frac{(\mp i a)^{n}}{n !} f^{(n)}(0) .
$$

For the distribution to be well-defined, this sum must converge. This means that the derivatives of $f$ must not grow too fast. In fact, if the series (2) is convergent, then the Taylor expansion for $f(x)$ around the point $x=0$ is convergent for $|x|<a$. Similarly, if we consider momentum-space distributions of the form $e^{i b p \pm a p}$ and require that their Fourier transform be well-defined, we will find that the Taylor expansion for $f(x)$ must converge for $|x-b|<a$. Hence, if $b$ is allowed to be arbitrary, then the test functions in $x$-space must be real-analytic. Moreover, they can be continued analytically off the real axis to a strip of width $a$.

The above arguments can be extended to the general $d$-dimensional case. We will see that if distributions grow exponentially in momentum space, the test functions in $x$-space must be real-analytic. An operator smeared with such a test function is not a local observable. Furthermore, the microlocality condition does not make any sense for such fields. Indeed, it says that if $f$ and $g$ are test functions whose supports are causally disconnected, then $\phi(f)$ and $\phi(g)$ commute (or anticommute). This condition is empty for quasilocal theories, since there are no test functions whose supports are causally disconnected.

One can nevertheless formulate a version of the microlocality axiom which does make sense. To formulate this axiom, we first need to define approximately local (AL) observables. The basic idea is to consider a sequence of test functions $\left\{f_{\nu}\right\}$ which get more and more concentrated on a certain compact set $M$. (The precise definition of what "concentrated" means will be given later.) Then we say that observables $\phi\left(f_{\nu}\right)$ are approximately local, and $M$ is their quasisupport. Thus quasisupport is an attribute of a sequence of observables rather than of a single observable. The quasilocality axiom says that AL observables whose quasisupports are space-like separated approximately commute (or anticommute). This means that if $M$ and $N$ are space-like separated, and $\phi\left(f_{\nu}\right)$ and $\phi\left(g_{\nu}\right)$ are sequences of AL observables with quasisupports $M$ and $N$, respectively, then

$$
\left[\phi\left(f_{\nu}\right), \phi\left(g_{\nu}\right)\right]_{-} \rightarrow 0 \text { or }\left[\phi\left(f_{\nu}\right), \phi\left(g_{\nu}\right)\right]_{+} \text {as } \nu \rightarrow \infty \text {. }
$$

The quasilocality axiom ensures locality "in the large." Nonlocal QFTs which satisfy this axiom are called quasilocal. Our proposal is that LSTs are quasilocal field theories.

The outline of the paper is as follows. In Sec. II we summarize the known properties of little string theories and argue that they cannot be local QFTs. In Sec. III we explain 
how the axioms of local quantum field theory should be modified in order to incorporate Wightman functions which grow exponentially in momentum space and why this leads to violations of locality at short distances. In Sec. IV we discuss how to define approximately local observables in nonlocal theories. It turns out that nontrivial observables approximately localized on a compact set $M$ can be defined for a special class of such theories, and only if $M$ is big enough. The precise definition of "big enough" sets depends on the reference frame. The minimal size of the support of an observable sets the smallest distance which observables in a quasilocal theory can probe. We argue that for little string theories this distance is of order $\sqrt{N} / M_{s}$. In Sec. V we suggest directions for future work.

\section{PROPERTIES OF LITTLE STRING THEORIES}

Let us summarize what is known about LSTs in general.

(1) LSTs are quantum-mechanical theories. This means that a state in LST is a ray in a Hilbert space $V$, and observables are self-adjoint linear operators on $V$. Even in ordinary quantum field theory many important observables (energy, for example) are unbounded operators which are only defined on a dense subset of $V$. Presumably the same is true about observables in LSTs.

(2) LSTs are Poincare-invariant, i.e. there is a unitary representation of the Poincaré group acting on $V$. In particular, there is a Hamiltonian (the generator of time translations) which is an unbounded self-adjoint operator on a dense subset of $V$. Furthermore, this operator has nonnegative spectrum. For all known LSTs this holds because they are supersymmetric.

(3) Among observables of LSTs there are operators $\phi_{R}(p)$ labeled by an irreducible finite-dimensional representation $R$ of $\operatorname{Spin}(1,5)$ and the "momentum" $p \in \mathbb{R}^{1,5}$ [14]. These operators can be thought of as functions on $\mathbb{R}^{1,5}$ valued in the tensor product $E n d(V) \otimes R$. They are covariant with respect to the Poincaré group, i.e. for any element $(a, \Lambda), a$ $\in \mathbb{R}^{1,5}, \Lambda \in S O(1,5)$, of the Poincare group we have

$$
U(a, \Lambda) \phi_{R}(p) U(a, \Lambda)^{-1}=e^{i p a} R(\Lambda) \phi\left(\Lambda^{-1} p\right) .
$$

(4) The Hilbert space $V$ has a distinguished state $\Omega$ (vacuum) which is Poincaré-invariant. Vacuum expectation values

$$
\begin{aligned}
\left(\Omega, \phi_{R_{1}}(p) \ldots \phi_{R_{n}}\left(p_{n}\right) \Omega\right) \sim & \delta^{6}\left(p_{1}+\ldots+p_{n}\right) \\
& \times \widetilde{W}_{n}\left(p_{1}-p_{2}, \ldots, p_{n-1}-p_{n}\right)
\end{aligned}
$$

appear to grow exponentially for large momenta. For example, the 2-point function seems to be growing as $[15,16]$

$$
\widetilde{W}_{2}(p) \sim \exp \left(\frac{c M_{s} p}{\sqrt{N}}\right)
$$

where $N$ is the number of 5-branes, and $c$ is a numerical constant of order 1. (The authors of [16] chose to remove this exponential growth by making a multiplicative renormalization of operators in momentum space. However, it appears that the growth is common to all correlators and is related to the growth of the density of states, see property (7). Multiplicative renormalization of fields is not sufficient to make higher-order correlators polynomially bounded, except in the case of a Gaussian theory.)

Strictly speaking, Eq. (6) has been established only for large $N$ and for momenta in the range $M_{s} / \sqrt{N} \ll p \ll M_{s}$. However, it is plausible that this growth continues for $p$ $\gg M_{s}$ [see property (7) below].

(5) Operators in LSTs obey the usual spin-statistics relation.

(6) In the infrared LSTs flow to local quantum field theories.

(7) The density of states of an LST grows exponentially at large energies [11]. Equivalently, the entropy per unit volume of the microcanonical ensemble is

$$
s \sim \frac{\epsilon \sqrt{N}}{M_{s}}
$$

if the energy density $\epsilon$ is large, $\epsilon \gg M_{s}^{6}$. Consequently, the canonical ensemble is defined only for temperatures $T$ $<M_{s} / \sqrt{N}$.

(8) An LST on a manifold of the form $\mathbb{R}^{1,5-n} \times T^{n}$ where $T^{n}$ is an $n$-dimensional torus with a flat metric is equivalent to a (in general different) LST with the same $M_{s}$ on a manifold $\mathbb{R}^{1,5-n} \times \hat{T}^{n}$ [9]. Here $\hat{T}^{n}$ is the dual torus. This means that different little string theories are related by $T$ dualities when compactified on tori. For example, the LST of parallel type IIA 5-branes is mapped by a T duality to the LST of parallel type IIA or IIB 5-branes, depending on whether $n$ is even or odd.

(9) Some further properties of LSTs are discussed in [1719].

Property (8) is particularly striking. One's first reaction is that a local quantum field theory cannot have a $T$ duality, and that only a string theory of some kind would fit the bill.

The former claim can be argued as follows. A local quantum field theory has local observables associated to compact sets. According to the microlocality axiom, these observables commute at space-like separations. Thus by looking at the structure of the algebra of observables one can reconstruct unambiguously the causal structure of space-time, i.e. the position of light-cones. In other words, one can reconstruct the conformal structure of space-time. Since in general a flat torus and its dual are not conformally equivalent, a local quantum field theory cannot have $T$ duality.

The claim that only a string theory can enjoy $T$ duality also sounds plausible. However since the only string theory we know of is critical string theory, and there is no agreement on how to define "string theory in general," this claim is almost devoid of content. We will argue below that little string theories are a kind of quasilocal field theories which do not have truly local observables. 


\section{LITTLE STRING THEORIES AS QUASILOCAL FIELD THEORIES}

\section{A. The space of test functions}

In local quantum field theory fields are operator-valued distributions [20]. This means that although the value of a field at a point is not a well-defined observable, the field smeared with a test function $f \in \mathcal{S}$ is well-defined. Usually $\mathcal{S}$ is taken to be the space of infinitely differentiable functions which decay at infinity faster than any negative power (the Schwartz space). The corresponding distributions (i.e. continuous linear functionals in the standard topology on $\mathcal{S}$ ) are called tempered distributions. Thus in local quantum field theory local fields are operator-valued tempered distributions.

The choice of the space of test functions seems like a technicality, but in fact it has important physical consequences. For example, since the Fourier transform of a function $f \in \mathcal{S}$ is again an element of $\mathcal{S}$ [21], tempered distributions can grow at most as a polynomial in momentum space. This implies that the correlators of local operators can grow at most as a power of momentum. If we want to accommodate operators whose matrix elements grow faster than a polynomial, one has to work with more singular distributions, which are defined on a smaller space of test functions.

In view of properties (4) and (7), it seems very likely that Wightman functions (i.e. vacuum expectation values) of operators $\phi_{R}(p)$ in little string theories grow exponentially with momenta. More precisely, by positivity of energy the function $\widetilde{W}_{n}\left(q_{1}, \ldots, q_{n-1}\right)$ vanishes when any of its arguments is outside the forward light-cone $V_{+}$, but inside $V_{+}^{n-1}$ it appears to be bounded by a multiple of

$$
\exp \left(+l\left(\left|q_{1}\right|+\ldots\left|q_{n-1}\right|\right)\right),
$$

where $|q|=\sqrt{q^{2}}$, and $l$ is of order $\sqrt{N} / M_{s}$. Clearly, such functions are not tempered distributions. ${ }^{1}$ Our first task is to find the right space of test functions which could be used to smear $\widetilde{W}_{n}$.

A necessary requirement on the test functions is that they be infinitely differentiable and decayed exponentially fast (in momentum space). The former requirement is necessary if we want the product of the field operator $\phi_{R}(x)$ and a polynomial of $x$ to be well-defined. The latter requirement comes from the exponential growth of the Wightman functions in momentum space. We also want the space of test functions to be Lorenz-invariant. Finally, the space of test functions should to be sufficiently "nice." As a minimum, we want it to be a complete countably normed space in which the nuclear theorem holds, see e.g. [20].

A convenient class of spaces of test functions was defined by Jaffe [22]. Given a function $g(t), t \in \mathbb{R}$, such that $g\left(t^{2}\right)$ is

\footnotetext{
${ }^{1}$ It is tempting to call them ill-tempered distributions, but probably the name "distributions of exponential growth" is more suitable.
}

entire $^{2}$ and positive, Jaffe defines a space $\widetilde{S}_{g}$ which consists of all functions on $\mathbb{R}^{d}$ which are infinitely-differentiable and for which all the norms

$$
\begin{gathered}
\|f(p)\|_{n}=\sup _{p ;|m| \leqslant n} g\left(n\|p\|^{2}\right)\left|D^{m} f(p)\right|, \\
n=1,2, \ldots,
\end{gathered}
$$

are finite. Here $m=\left(m_{1}, \ldots, m_{d}\right)$ is a polyindex, $|m|$ $=\sum_{i} m_{i}$, and $\|p\|$ is an arbitrary Euclidean norm on $\mathbb{R}^{d}$. Loosely speaking, the finiteness of the norms (9) means that a test functions and all its derivatives decay at infinity faster than $1 / g\left(n\|p\|^{2}\right)$ for any positive $n$. Despite appearances, $\widetilde{S}_{g}$ does not depend on the choice of the Euclidean norm, but it does depend on the rate of growth of $g\left(t^{2}\right)$ at infinity. If we define convergence on $\widetilde{S}_{g}$ using the family of norms (9), it becomes a complete countably normed space. It is easy to see that $\widetilde{S}_{g}$ is Lorenz-invariant. In addition, if $g$ satisfies

$$
\begin{gathered}
\frac{g\left(n\|p\|^{2}\right)}{g\left(n^{\prime}\|p\|^{2}\right)} \text { is an integrable function for all } n \\
\text { and sufficiently large } n^{\prime},
\end{gathered}
$$

the nuclear theorem holds [21].

Let us denote the Fourier transform of $\widetilde{S}_{g}$ by $S_{g}$. We think of $\widetilde{S}_{g}$ as the space of test functions in momentum space, so $S_{g}$ is the space of test-functions in $x$-space. The spaces $S_{g}$ can be used to define quantum field theories whose ultraviolet behavior is more singular than that of Wightman QFTs. Their localizability properties depend on the rate of growth of $g$ at infinity.

A. Jaffe showed [22] that if the function $g$ satisfies

$$
\int_{0}^{\infty} \frac{\log g\left(t^{2}\right)}{1+t^{2}} d t<\infty
$$

then the Fourier transform of $\widetilde{S}_{g}$ contains many functions with compact support, so that the microlocality axiom can be formulated in the usual manner. QFTs based on the space $S_{g}$ with $g$ satisfying Eq. (11) are called strictly localizable [22]. Such QFTs have properties which are not very different from the properties of Wightman QFTs.

Conversely, if $g$ does not satisfy Eq. (11), there are no functions with compact support among the test functions in $x$-space (except identical zero). For little string theories we want to take

$$
g(t)=e^{\sqrt{t}}
$$

\footnotetext{
${ }^{2} \mathrm{By}$ an entire function on $\mathbb{R}^{m}$ we mean a real-analytic function whose Taylor series has an infinite radius of convergence. An entire function can be analytically continued to a holomorphic function on $\mathrm{C}^{m}$.
} 
This choice of $g$ is dictated by the exponential growth of the Wightman functions (8). With this choice of $g$ the test functions in momentum space decrease faster than any linear exponential, thus ensuring that the value of the Wightman functional is well-defined on any $n$-tuple

$$
\left(f_{1}, \ldots, f_{n}\right), \quad f_{1}, \ldots, f_{n} \in \widetilde{S}_{g} .
$$

Since the condition (11) is violated, the test functions in $x$-space cannot have compact support. In fact, one can show that the test functions in $x$-space are entire. An entire function which vanishes in an open set is identically zero; in particular the support of any nontrivial entire function is the whole $\mathbb{R}^{d}$.

\section{B. Analytic properties of Wightman functions in LSTs}

In this section we discuss the analyticity properties of Wightman functions in LSTs, assuming that LSTs are quantum field theories based on the Jaffe space $S_{g}$ with $g(t)$ $=\exp (\sqrt{t})$. Our motivation is the following. Recall that in local QFTs Wightman functions obey certain symmetry properties as a consequence of microlocality, and conversely microlocality follows from these symmetry properties $[20,23]$. One possible way to ensure locality of LSTs "in the large" is to impose a similar symmetry requirement on their Wightman functions. However, before we do this, we need to understand at which points the values of Wightman functions are well-defined. After all, "Wightman functions" are really distributions, and pretty singular ones at that. Luckily, this problem was studied in detail in the literature on nonlocal field theories. Below we summarize some of this work following [24].

In a local quantum field theory the microlocality axiom implies the permutation symmetry of Wightman functions,

$$
\begin{aligned}
& W_{n}\left(x_{1}, \ldots, x_{i}, x_{i+1}, \ldots, x_{n}\right) \\
& \quad=W_{n}\left(x_{1}, \ldots, x_{i+1}, x_{i}, \ldots, x_{n}\right),
\end{aligned}
$$

for $\left(x_{i}-x_{i+1}\right)^{2}<0$. (To simplify our discussion, we will restrict ourselves to bosonic fields here.) Since "Wightman functions" are not really functions, their values at points are not well-defined in general, and the meaning of Eq. (13) must be clarified.

One way to interpret Eq. (13) is to smear it with a product of test functions

$$
f_{1}\left(x_{1}\right) \ldots f_{n}\left(x_{n}\right)
$$

such that the supports of $f_{i}$ and $f_{i+1}$ are space-like separated. However this approach does not extend to QFTs with entire test functions. A more sophisticated interpretation of Eq. (13) makes use of the fact that at certain special points the values of Wightman functionals are well-defined. Recall that Wightman functionals can be regarded as boundary values of certain holomorphic functions [20]. In other words, one can define "analytic continuation" of the n-point Wightman functional to complex values of its arguments, and this continuation is a holomorphic function in a certain open set $\mathcal{T}_{n} \subset \mathrm{C}^{d n} . \mathcal{T}_{n}$ is called the forward tube and is given by

$$
\mathcal{T}_{n}=\left\{x+i y \mid x, y \in \mathbb{R}^{d n} ; \zeta_{i}(y) \in V_{+}, i=1, \ldots, n-1\right\} .
$$

Here $V_{+}$is the forward light-cone in $\mathbb{R}^{d}$, and for any point $\left(x_{1}, \ldots, x_{n}\right) \in \mathbb{R}^{d n}$ we denote $\zeta_{i}(x)=x_{i}-x_{i+1}, i=1, \ldots, n$ -1 . The real points are on the boundary of $\mathcal{T}_{n}$. The possibility of "analytic continuation" is a consequence positivity of energy in local quantum field theory, see [20] for details. (Really, "analytic continuation" is simply the Laplace transform of momentum space Wightman functions which is well-defined because momentum space Wightman functions vanish outside the forward light-cone.) If we apply complexified Lorenz transformations to the forward tube $\mathcal{T}_{n}$, we get the so-called extended forward tube $\mathcal{T}_{n}^{e x t}$. By Lorenzinvariance, the "analytic continuations" of Wightman functionals are holomorphic in the extended forward tube [20]. Now it is crucial that $\mathcal{T}_{n}^{\text {ext }}$ includes real points (usually called Jost points). Jost points form an open set which we will call the Jost domain. Wightman functionals can be regarded as ordinary functions at all Jost points. When both

$$
\left(x_{1}, \ldots, x_{i}, x_{i+1}, \ldots, x_{n}\right)
$$

and

$$
\left(x_{1}, \ldots, x_{i+1}, x_{i}, \ldots, x_{n}\right)
$$

belong to the Jost domain, Eq. (13) admits a straightforward interpretation.

Which real points belong to the Jost domain? The answer it simple for a 2-point function: $\left(x_{1}, x_{2}\right)$ is a Jost point if and only if $\left(x_{1}-x_{2}\right)^{2}<0$. Thus the 2-point Wightman function is analytic for space-like separated points. Equation (13) simply says that $W_{2}$ is a symmetric function of its arguments when its arguments are space-like separated. The situation for the $n$-point functions is similar, except that the shape of the Jost domain is somewhat more complicated [20]: it is not sufficient to require that all vectors $x_{i}-x_{j}$ be space-like, one should also require that every point in the convex hull of the points $\zeta_{i}(x)=x_{i}-x_{i+1}, i=0, \ldots, n-1$ be space-like. This condition is also a necessary one for $\left(x_{1}, \ldots, x_{n}\right)$ to belong to the Jost domain.

One can prove (see e.g. [23]) that the permutation symmetry of Wightman functionals in the Jost domain is equivalent to microlocality, if the Wightman functionals are assumed to be tempered.

After this brief review of the analytic properties of Wightman functions in a local QFT, let us return to QFTs based on the space $S_{g}$ with $g(t)=\exp (\sqrt{t})$. The analytic properties of Wightman functions in these theories were described in [24]. For a given Wightman function one can define the analogue of the forward tube $\mathcal{U}_{n}$ as the region in $\mathrm{C}^{d n}$ where the Laplace transform of the momentum-space Wightman function is well-defined. It turns out that this domain is given by

$$
\mathcal{U}_{n}=\left\{x+i y \mid x \in \mathbb{R}^{d n} ; y \in \underset{\eta}{\cup} V_{l}^{n}(\eta)\right\} .
$$

Here $l$ is a positive real number, $\eta \in \mathbb{R}^{d}$ is a unit time-like vector, and $V_{l}^{n}(\eta)$ is a domain in $\mathbb{R}^{d n}$ given by 


$$
\begin{aligned}
V_{l}^{n}(\eta)= & \left\{\left(x_{1}, \ldots, x_{n}\right) \in \mathbb{R}^{d n} \mid \zeta_{i}(x) \cdot \eta\right. \\
& \left.-\sqrt{\left(\zeta_{i}(x) \cdot \eta\right)^{2}-\zeta_{i}(x)^{2}}>l, \forall i\right\} .
\end{aligned}
$$

The meaning of the above equation is easiest to see in the frame where $\eta=(1, \overrightarrow{0})$. Then $V_{l}^{n}(\eta)$ becomes

$$
V_{l}^{n}(\eta)=\left\{\left(x_{1}, \ldots, x_{n}\right) \in \mathbb{R}^{d n}\left|\zeta_{i 0}(x)-\right| \vec{\zeta}_{i}(x) \mid>l, \forall i\right\} .
$$

It is easy to see that for $l>0$ the domain $\mathcal{U}_{n}$ is a proper subset of $\mathcal{T}_{n}$, and in the limit $l \rightarrow 0$ the two domains coincide.

The parameter $l$ can be different for different Wightman functions and characterizes the nonlocality of a given Wightman function. For $l>0$ the closure of $\mathcal{U}_{n}$ does not include real points, unlike in a local QFT. Thus Wightman functions in $x$-space are not boundary values of holomorphic functions, in general.

As before, Lorenz invariance implies that the Wightman functions are analytic in the domain $\mathcal{U}_{n}^{e x t}$ obtained by applying complexified Lorenz transformations to $\mathcal{U}_{n}$. The Jost domain is defined as the set of real points of $\mathcal{U}_{n}^{\text {ext }}$.

It is of interest to determine the precise shape of the Jost domain in the nonlocal case. This has been done in [24]. It is rather obvious that for $l>0$ the "nonlocal" Jost domain is a proper subset of the "local" Jost domain. For $n=2$ the "nonlocal" Jost domain is given by

$$
\left(x_{1}-x_{2}\right)^{2}<-l^{2} \text {. }
$$

For $n>2$ a natural guess for what a "nonlocal" Jost domain is the following. Let $J_{n}^{l}$ be the set of all points $x \in \mathbb{R}^{n d}$ such that the convex hull of $\zeta_{1}(x), \ldots, \zeta_{n-1}(x)$ belongs to the hyperboloid $\zeta^{2}<-l^{2}$. For $l \rightarrow 0 \quad J_{n}^{l}$ reduces to the "local", Jost domain, and is a natural candidate to be the "nonlocal", Jost domain. This guess is incorrect. The actual shape of the "nonlocal"' Jost domain is rather more complicated, see [24] for details. For our purposes it is sufficient to know that the "nonlocal" Jost domain is a proper subset of $J_{n}^{l}$ [24]. Since the closure of $J_{n}^{l}$ does not contain any light-like separated points, the same is true about the "nonlocal" Jost domain. This means that in nonlocal field theories Wightman functions may have singularities outside the light-cone. We will see some examples of this below.

In view of the above, there is an obvious reformulation of the microlocality axiom which also makes sense for nonlocal field theories based on the space $S_{g}$. We simply require (anti)symmetry of Wightman functions in their "nonlocal" Jost domains. We will call this symmetry requirement weak quasilocality (strong quasilocality will be discussed in the next section). Nonlocal field theories whose Wightman functions satisfy the weak quasilocality axiom will be called weakly quasilocal.

In a general weakly quasilocal field theory $l$ can grow without bound as the order of the Wightman function increases. Then the theory is nonlocal at all scales, if one studies a sufficiently complicated Wightman function. In little string theories the exponential growth of Wightman functions is caused by the exponential growth of the density of states. Thus it is natural to suspect that in LSTs $l$ is bounded from above by a quantity of order $\sqrt{N} / M_{s}$. In other words, LSTs are probably "more local" than a generic weakly quasilocal field theory. In Sec. IV we will argue that LSTs satisfy a stronger condition (strong quasilocality) which implies weak quasilocality, boundedness of $l$, as well as the existence of approximately local observables.

\section{A sample 2-point function}

Let us illustrate the preceding discussion with a simple example of a 2-point function of a real scalar field. Poincaréinvariance and positivity of energy imply that the most general 2-point function in momentum space has the form

$$
\widetilde{W}_{2}(p)=\theta\left(p_{0}\right) \theta\left(p^{2}\right) \sigma\left(p^{2}\right),
$$

where the function $\sigma$ is positive and measurable. $\sigma(t)$ is the density of states times some form factor. In a local QFT $\sigma$ cannot grow faster than a polynomial, but in a weakly quasilocal QFT we only require that $\sigma(t)$ be bounded by $\exp (l \sqrt{t})$ for some $l$. In particular, we know that in LSTs the density of states grows like $\exp (l E)$ with $l$ of order $\sqrt{N} / M_{s}$. If the form factor does not decrease exponentially, the function $\sigma(t)$ will grow like $\exp (l \sqrt{t})$. Computation in $[16,15]$ seems to support this conjecture.

As mentioned above, the Jost domain for a 2-point function is

$$
\left(x_{1}-x_{2}\right)^{2}<-l^{2}
$$

The weak quasilocality condition is satisfied automatically, because $W_{2}(x)$ is a function of $x^{2}$ by Lorenz invariance.

Given that the Wightman functional is a symmetric function of its arguments in the region (20) essentially by virtue of Lorenz invariance, one may ask how it can fail to be symmetric for all space-like points. The answer is very simple. In a quasilocal theory the Wightman function may have poles in the region $-l^{2} \leqslant\left(x_{1}-x_{2}\right)^{2}<0$. Thus one needs a prescription how to treat these poles. The correct prescription involves factors like $\operatorname{sgn}\left(t_{1}-t_{2}\right)$, where $t_{1}, t_{2}$ are the time-like components of $x_{1}, x_{2}$. Such factors are Lorenz-invariant, but not symmetric under the exchange of $x_{1}$ and $x_{2}$.

To illustrate this point, let us consider the following simple example in $d=4$ borrowed from [24]. Set

$$
\sigma=e^{l \sqrt{t}} / \sqrt{t}
$$

The Fourier integral

$$
W_{2}(z)=\int d^{4} p e^{-i p z} \widetilde{W}_{2}(p)
$$

converges in the region $(\operatorname{Im} z)^{2}>l^{2}$. In this region we get

$$
W_{2}(z)=\frac{1}{2 \pi^{2}\left(z^{2}+l^{2}\right)}\left\{\frac{2 l}{z^{2}}+\frac{1}{\sqrt{z^{2}+l^{2}}} \log \frac{l+\sqrt{l^{2}+z^{2}}}{l-\sqrt{l^{2}+z^{2}}}\right\} .
$$


If we formally continue $W_{2}(z)$ to real values of $z$, we find a pole at $z^{2}=-l^{2}$ and a branch point at $z^{2}=0$. The branch point at $z^{2}=0$ is usually present in a local quantum field theory as well, but the pole at space-like $z$ is possible only in a nonlocal theory. Because of this pole, the integral

$$
\int_{\operatorname{Im} z=0} d^{4} z W_{2}(z) f(z)
$$

is not well-defined. To derive the right prescription, we recall that $f(z)$ is an entire function, and replace the integral above by

$$
\int_{\operatorname{Im} z=\eta} d^{4} z W_{2}(z) f(z) .
$$

If $\eta^{2}>l^{2}$, the integral is well-defined. Deforming the integration contour back to the real subspace, we find the prescription for treating the pole in Eq. (23):

$$
\frac{1}{z^{2}+l^{2}} \rightarrow \lim _{\epsilon \rightarrow 0} \frac{1}{z^{2}+l^{2}+i \epsilon z \eta}
$$

The latter distribution is Lorenz-invariant, but not invariant under $z \rightarrow-z$.

There is a well-known theorem (the theorem about global nature of local commutativity, see e.g. [23]) which, roughly speaking, says that if the fields (anti)commute for large enough space-like separations, then they (anti)commute for all space-like separations. This statement, when rephrased in terms of Wightman functions, is clearly invalid in the above example. The theorem about the global nature of local commutativity does not apply because one of the assumptions in its proof is that the Wightman functions are tempered distributions.

\section{APPROXIMATELY LOCAL OBSERVABLES IN LSTS}

\section{A. General idea}

In the previous section we saw that operators in little string theories should be smeared with entire test functions. Since a nontrivial entire function cannot vanish in an open set, the corresponding observables are highly nonlocal. On the other hand, at low energies LSTs flow to local quantum field theories [property (6)]. Thus the theories should be approximately local at distances larger than $\sqrt{N} / M_{s}$. As explained in the Introduction, the microlocality axiom is empty if only analytic test functions are allowed, so we need to find some replacement for it which would ensure the locality of LSTs "in the large."

The weak quasilocality postulate discussed in the previous section ensures that Wightman functions are symmetric functions of their arguments when the arguments are far apart and space-like separated. However, in order to be able to claim that a nonlocal theory flows to a local theory in the infrared, one needs more than this. A local theory has local observables associated to compact sets. If a nonlocal theory is approximately local in the infrared, there should be a way to define approximately local (AL) observables associated to compact sets which are "large" in some sense. Furthermore, one should require that AL observables approximately commute (or anticommute) when their supports are space-like.

In the case of little string theories, we know for a fact that they flow to local quantum field theories in the infrared, so understanding approximately local observables is of paramount importance.

The problem of defining AL observables in nonlocal field theories was previously addressed in $[25,26,13]$. The present section is our interpretation of [13]. We assume that the reader is reasonably comfortable with the notion of a topological vector space. Readers with low tolerance for math may skip this section on first reading; such readers should be warned that the discussion below touches on some important physics of LSTs.

To define observables approximately localized on a closed set $M \subset \mathbb{R}^{d}$, it is natural to consider a sequence of test functions $\left\{f_{\nu}\right\}$ which converges to zero in the open set $\mathrm{R}^{d} \backslash M$ in some sense. Then observables $\phi\left(f_{\nu}\right)$ should be regarded as approximately localized in $M$, the approximation getting better as $\nu$ increases. To define "convergence in an open set," we need a topology $\tau(\mathcal{O})$ on the space of test functions for each open set $\mathcal{O} \subset \mathbb{R}^{d}$. The meaning of $\tau(\mathcal{O})$ is the following: two test functions are "close", in the topology $\tau(\mathcal{O})$ if and only if they are "close", everywhere in $\mathcal{O}$. We then say that a sequence of test functions is localized on a closed set $M$ if it is convergent to zero in the topology $\tau\left(\mathbb{R}^{d} \backslash M\right)$. We will also say that $M$ is a quasisupport of the sequence $\left\{f_{\nu}\right\}$.

The main problem is how to choose the topologies $\tau(\mathcal{O})$. A natural restriction on the choice of topologies is that if $\mathcal{O}_{1} \subset \mathcal{O}_{2}$, then $\tau\left(\mathcal{O}_{1}\right)$ should be weaker than $\tau\left(\mathcal{O}_{2}\right)$. In other words, if two functions are "close" on $\mathcal{O}_{2}$, they should be "close" on $\mathcal{O}_{1}$. Another natural restriction is to require that $\tau\left(R^{n}\right)$ be the same as the original topology on the space of test functions. Indeed, a sequence $\left\{f_{\nu}\right\}$ converging to zero in the topology $\tau\left(\mathbb{R}^{n}\right)$ should be regarded as approximating a function which is identically zero. Then it is natural to require that $\phi\left(f_{\nu}\right)$ converge to zero. Thus all fields must be continuous functionals in the topology $\tau\left(\mathbb{R}^{n}\right)$. The original topology on $S_{g}$ has this property by definition, and in general there is no other natural topology with this property.

What is the "original" topology on $S_{g}$ ? One way to define it is to use the family of norms (9) to define convergence on $\widetilde{S}_{g}$ and then apply Fourier transform. We are going to use an equivalent definition $[21,13]$ which makes use of the fact that all functions in $S_{g}$ can be analytically continued to $\mathrm{C}^{d}$. The topology on $S_{g}$ can be specified by saying which sequences of functions converge to zero. We declare that a sequence $\left\{f_{\nu}\right\}$ converges to zero in the topology $\tau\left(\mathbb{R}^{n}\right)$, if it converges to zero uniformly in all sets of the form

$$
V_{a}=\left\{x+i y \mid x, y \in \mathbb{R}^{d},\|\operatorname{Im} y\| \leqslant a\right\}, \quad a>0 .
$$

One can check that with this choice of topology $S_{g}$ becomes a complete countably normed Montel space [21].

If we do not assume anything about the quasilocal theory in question, then the only natural choice for $\tau(\mathcal{O})$ seems to 
be the topology of uniform convergence in all sets of the form

$$
V_{a}(\mathcal{O})=\left\{x+i y \mid x \in \mathcal{O}, y \in \mathbb{R}^{d},\|\operatorname{Im} y\| \leqslant a\right\}, \quad a>0 .
$$

This family of topologies satisfies both of the above requirements.

However, despite appearances, this choice of $\tau(\mathcal{O})$ does not really allow to define nontrivial observables associated to compact sets. Indeed, consider a compact set $M$, and a sequence of functions converging to zero in the topology $\tau\left(\mathbb{R}^{d} \backslash M\right)$. According to our definition, we say that such a sequence of functions is localized on $M$. However, it turns out that any such sequence actually converges uniformly to zero everywhere. (The proof of this fact is very simple and is left as an exercise for the reader. See also [13], Sec. 1.8, where a similar statement is proven.) This means that if $M$ is any compact closed set, $\tau\left(\mathbb{R}^{d} \backslash M\right)$ coincides with $\tau\left(\mathbb{R}^{d}\right)$. Consequently, this family of topologies does not allow to tell apart different compact sets of $\mathbb{R}^{d}$, or even to tell apart a compact set from the empty set.

The lesson here is that entire test functions are too smooth to allow a sensible definition of quasisupport.

\section{B. Further constraints on the ultraviolet behavior of fields}

To do better than this, we need to impose some additional constraints on the high-energy behavior of fields. To motivate these constraints, we first define a new space of test functions $\widetilde{S}_{\eta}^{l}$, where $\eta$ is a unit time-like vector and $l>0$ is a number. $\widetilde{S}_{\eta}^{l}$ consists of all infinitely differentiable functions on $\mathrm{R}^{d}$ all of whose derivatives decay faster than

$$
\exp \left(-l\|p\|_{\eta}\right)
$$

Here $\|p\|_{\eta}^{2}=2(p \cdot \eta)^{2}-p^{2}$ is a Euclidean norm on $\mathbb{R}^{d}$ associated with the vector $\eta$. The space $\widetilde{S}_{\eta}^{l}$ was first introduced by Shilov [27] and studied in detail in [21]. With a natural choice of topology $\widetilde{S}_{\eta}^{l}$ becomes a complete countably normed Montel space, just like $\widetilde{S}_{g}$.

Obviously, if $l>l^{\prime}$, then $\widetilde{S}_{\eta}^{l} \subset \widetilde{S}_{\eta}^{l^{\prime}}$. Thus for any fixed $\eta$, we have an infinite decreasing sequence of spaces

$$
\widetilde{S}_{\eta}^{1} \supset \widetilde{S}_{\eta}^{2} \supset \widetilde{S}_{\eta}^{3} \supset \ldots
$$

It is easy to see that our basic space of momentum-space test functions $\widetilde{S}_{g}$ is the intersection of all these spaces:

$$
\widetilde{S}_{g}=\bigcap_{l=1,2, \ldots} \widetilde{S}_{\eta}^{l}
$$

Moreover, one can check that the standard topology on $\widetilde{S}_{g}$ is the direct limit of the standard topologies on $\widetilde{S}_{\eta}^{l}$.

The Fourier transform of $\widetilde{S}_{\eta}^{l}$ will be denoted $S_{\eta}^{l}$. According to [21], $S_{\eta}^{l}$ consists of functions which decay faster than any polynomial at infinity and can be continued analytically into a strip

$$
\left\{x+i y \mid x, y \in \mathbb{R}^{d},\|\operatorname{Im} y\|_{\eta}<l\right\} .
$$

The crucial difference between $S_{\eta}^{l}$ and $S_{g}$ is that in the latter case all test functions are entire, while in the former case they can only be continued into a strip of width $l$ off the real slice $\mathbb{R}^{d} \subset \mathbb{C}^{d}$.

Our basic space of test functions $S_{g}$ is the direct limit of spaces $S_{\eta}^{l}, l=1,2, \ldots$ The standard topology on $S_{\eta}^{l}$ is the topology of uniform convergence in all sets of the form

$$
V_{a}^{\eta}=\left\{x+i y \mid x, y \in \mathbb{R}^{d},\|\operatorname{Im} y\|_{\eta} \leqslant a\right\}, \quad 0<a<l .
$$

The space $S_{\eta}^{l}$ is not Lorenz-invariant and so cannot be used as the basic space of test functions in a QFT. Nevertheless these spaces play an important role in QFTs based on the space $S_{g}$. Namely, one can show that any matrix element of $\phi(x)$ is a distribution on $S_{\eta}^{l}$ for some $l$ and $\eta[24,21]$. In general, $l$ depends on the states between which $\phi(x)$ is sandwiched.

In order to define approximately local observables, we will require that all fields be well-defined operator-valued distributions on $S_{\eta}^{l}$ for some fixed $l$ and $\eta$. Then by Lorenzinvariance all fields are well-defined distributions on $S_{\eta}^{l}$ for all $\eta$.

\section{Definition and properties of approximately local observables}

With this additional requirement it becomes possible to define AL observables associated to all compact sets of size bigger than $l$. More precisely, for any unit time-like vector $\eta$ we can define a family of topologies $\tau_{\eta}(\mathcal{O})$ which satisfies all the requirements stated above. Thus possible definitions of localization are labeled by $\eta$. It is tempting to interpret $\eta$ as the 4-velocity of a reference frame. Then we have a different notion of approximate localization for different frames.

It is clear what the definition of topologies $\tau_{\eta}(\mathcal{O})$ should be. We simply take $\tau_{\eta}(\mathcal{O})$ to be the topology of uniform convergence on all sets of the form

$$
V_{a}^{\eta}(\mathcal{O})=\left\{x+i y \mid x \in \mathcal{O}, y \in \mathbb{R}^{d},\|\operatorname{Im} y\|_{\eta} \leqslant a,\right\}, \quad 0<a<l .
$$

It is easy to see that both requirements on the family $\tau(\mathcal{O})$ stated above are satisfied.

We need to check now that this new family of topologies allows to tell apart different compact sets in $\mathbb{R}^{d}$. This problem was addressed in [13]. These authors showed that the family $\tau_{\eta}(\mathcal{O})$ can tell apart a compact set $M$ from the empty set only if $M$ is big enough. For example, let $M$ be a ball

$$
B_{a}=\left\{x \mid x \in \mathbb{R}^{d},\|x\|_{\eta} \leqslant a\right\} .
$$

If $a<l$, then it turns out that any sequence of functions localized on $M$ converges uniformly to zero everywhere on $\mathrm{R}^{d}$. In other words, for $a<l \tau\left(\mathbb{R}^{d} \backslash M\right)$ coincides with $\tau\left(\mathbb{R}^{d}\right)$, and there are no nontrivial observables localized on $M$ [13]. On the other hand, if $a \geqslant l$, then the topologies $\tau\left(\mathbb{R}^{d} \backslash M\right)$ and 
$\tau\left(\mathbb{R}^{d}\right)$ are different, and there are nontrivial observables approximately localized on $M[26,13]$.

One can give a criterion which determines if the compact set $M$ is "big enough." The key mathematical input is the notion of the domain of holomorphy [28]. We say that an open set $\Omega$ in $C^{d}$ is a domain of holomorphy if there exists a function $f$ which is holomorphic in $\Omega$ and cannot be analytically continued to a bigger open set. For any open set $X$ we define its domain of holomorphy $\Omega_{X}$ as the smallest domain of holomorphy containing $X$. If $X$ is itself a domain of holomorphy, then $\Omega_{X}=X$, otherwise $\Omega_{X}$ is strictly larger than $X$. Now let $M$ be a compact set in $\mathrm{R}^{d}$ and let us set

$$
X=\left\{x+i y\left|x \in \mathbb{R}^{d} \backslash M,\right| \mid \operatorname{Im} y \|_{\eta}<l\right\} .
$$

It may well happen that $\Omega_{X}$ is the whole strip (32). In this case $M$ is too small, in the sense that the topologies $\tau\left(\mathbb{R}^{d} \backslash M\right)$ and $\tau\left(\mathbb{R}^{d}\right)$ are equivalent. On the other hand, if $\Omega_{X}$ does not contain the whole strip (32), then convergence in the topology $\tau\left(\mathbb{R}^{d} \backslash M\right)$ does not imply convergence in the topology $\tau\left(\mathbb{R}^{d}\right)$, and nontrivial observables associated to $M$ exist $[26,13]$.

\section{The strong quasilocality axiom}

We have now defined the notion of AL observables (different for different reference frames). As discussed in Sec. I, if a theory is approximately local at long distances, AL observables must approximately commute if their quasisupports are space-like separated. More precisely, let $M$ and $N$ be two closed sets which are space-like separated, and let $\left\{f_{\nu}\right\}$ and $\left\{g_{\nu}\right\}$ be sequences of test functions (from the space $S_{\eta}^{l}$ ) whose quasisupports are $M$ and $N$ respectively. Then the strong quasilocality axiom states that

$$
\left[\phi\left(f_{\nu}\right), \phi\left(g_{\nu}\right)\right]_{-} \rightarrow 0 \text { or }\left[\phi\left(f_{\nu}\right), \phi\left(g_{\nu}\right)\right]_{+} \rightarrow 0 \text { as } \nu \rightarrow \infty .
$$

Nonlocal QFTs satisfying this axiom will be called "strongly quasilocal," or simply "quasilocal."

Our conjecture about the universality class of LSTs can now be stated very concisely. We propose that LSTs are strongly quasilocal QFTs in six dimensions, with $l$ of order $\sqrt{N} / M_{s}$.

It is shown in [13] that strong quasilocality implies weak quasilocality. Furthermore, it can be shown that weakly quasilocal QFTs obey the CPT and spin-statistics theorems [24]. This agrees with what we know about LSTs.

With additional assumptions, such as the existence of a mass gap, one can also define a unitary $S$-matrix in quasilocal QFTs and prove Froissart-type bounds on the cross sections $[29,13]$. However, LSTs do not have a mass gap, and consequently the $S$-matrix is not well-defined in these theories.

\section{E. An example of a quasilocal QFT}

Let us give a simple example of a QFT which satisfies the strong quasilocality axiom. Let $\phi(m, x)$ be a one-parameter family of scalar fields satisfying the commutation relation

$$
\left[\phi(m, x), \phi\left(m^{\prime}, x^{\prime}\right)\right]=\delta\left(m-m^{\prime}\right) \Delta_{m}\left(x-x^{\prime}\right),
$$

where $\Delta_{m}\left(x-x^{\prime}\right)$ is the commutator function of a free scalar field of mass $m$. In other words, for any $m$ the field $\phi(m, x)$ is a free scalar field of mass $m$, and these fields commute for different $m$ 's. We define

$$
\phi(x)=\int_{0}^{\infty} d m \sqrt{\sigma\left(m^{2}\right)} \phi(m, x),
$$

where $\sigma(t)$ is given by Eq. (21). Let $\Omega$ be the common vacuum for all $\phi(m, x)$. We define the Hilbert space of our theory as a completion of the space spanned by the vectors

$$
\phi\left(f_{1}\right) \phi\left(f_{2}\right) \ldots \phi\left(f_{n}\right) \Omega,
$$

where $f_{i}$ are suitable test functions (see below), and $n$ runs over all nonnegative integers.

Obviously, the field $\phi(x)$ is Gaussian: all its $n$-point function are expressible as products of its 2-point function $W_{2}\left(x-x^{\prime}\right)$ using Wick's theorem. $W_{2}\left(x-x^{\prime}\right)$ is given by the formulas (23),(25) and is a distribution on $S_{g}$ with $g(t)$ $=\exp (\sqrt{t})$. Thus we can take $S_{g}$ with $g(t)=\exp (\sqrt{t})$ as our space of test functions.

This Gaussian quasilocal theory is reminiscent of the toy model discussed in Appendix $\mathrm{C}$ of [16]. The toy model is a scalar field in $1+1$ dimensions whose mass undergoes a jump at $x=0$ but is otherwise free. This theory can be reinterpreted as a holographic dual of a certain quantummechanical boundary theory living at $x=0$. The boundary theory has a single operator $O(t)$ whose 2-point function grows exponentially in the energy representation, and whose higher-order connected correlators vanish. Our Gaussian QFT can be regarded as a higher-dimensional version of this boundary theory.

The commutator

$$
[\phi(x), \phi(y)]=W_{2}(x-y)-W_{2}(y-x)
$$

in our theory does not vanish outside the light cone, as explained in Sec. III C. Instead it has a contribution proportional to

$$
\delta\left((x-y)^{2}+l^{2}\right) \operatorname{sgn}\left(x_{0}-y_{0}\right) .
$$

It is easy to see that the 2-point momentum-space Wightman function (19) is well-defined as a distribution on $\widetilde{S}_{\eta}^{l}$ for any $\eta$. Since the theory is Gaussian, this immediately implies that the same is true for all higher-point functions. Hence the theory satisfies the strong quasilocality condition.

For an example of an interacting weakly quasilocal QFT, see $[30]$.

\section{DISCUSSION}

Hopefully, by relating little string theories to quasilocal quantum field theories, we have clarified the nature of the former, especially the degree to which observables in little string theories can be localized. We argued that there are no strictly local observables in LSTs, but there are observables 
which can be approximately localized (with arbitrary accuracy) to regions whose size is bigger than $\sqrt{N} / M_{s}$. Thus LSTs have a fundamental length scale which sets a limit on the resolution which can be achieved by measuring physical observables. Somewhat unexpectedly, the notion of localization depends on the reference frame: observers in different reference frames have different procedures for measuring approximately local observables. This is achieved by introducing a different space of test functions for each reference frame.

We also suggested that LSTs obey the strong quasilocality axiom which ensures that the theory is approximately local at large distances. This axiom is a surrogate for the usual microlocality axiom and preserves most of the usual consequences of microlocality ( $C P T$ invariance and the spinstatistics relation) $[24,13]$.

On general grounds, it appears very natural that little string theories do not have truly local observables. After all, interacting critical string field theory also appears to violate locality $[31,32]$. What is surprising (at least to the author) is that this nonlocality can be accommodated simply by choosing a space of test functions different from the usual Schwartz space $\mathcal{S}$.

We saw that many known properties of little string theories fit into the framework of quasilocal field theory with $l$ $\sim \sqrt{N} / M_{s}$. One important feature which we have not explained is $T$ duality. $T$ duality is related to the behavior of little string theory at distances of order $1 / M_{s}$. Presumably, only very special quasilocal field theories enjoy this property. At any rate, the fact that observables in quasilocal field theories cannot be localized to distances shorter than the fundamental scale suggests that $T$ duality is not impossible for field theories in this class.

In this paper we have focused on the kinematics of LSTs, but of course one would like to understand their dynamics as well. Since the usual definition of LSTs based on decoupling in critical string theory is very implicit, this is a hard problem. Some progress in this direction has been made in [1719]. Our hope is that the ultraviolet behavior of LSTs is simple enough. If this is the case, LSTs may provide some insight into $(2,0)$ and $(1,0)$ superconformal field theories to which they flow in the infrared.

If our proposal is correct, then $(1,1)$ LSTs realize the old idea that a nonrenormalizable quantum field theory may arise as the infrared limit of a quasilocal field theory. (This was the primary motivation for the study of quasilocal theories in 1960s and 1970s.) Indeed, consider a maximally supersymmetric super-Yang-Mills theory in six dimensions with a simply-laced gauge group. Such a theory is nonrenormalizable, and there seems to be no local quantum field theory which flows to it in the infrared. However, it emerges as the infrared limit of a certain quasilocal field theory, namely an LST with $(1,1)$ supersymmetry (SUSY) [9].

A more speculative proposal is to try to use quasilocal field theories to model nonlocality arising in critical string theory. In particular, we have in mind applications to the Hawking information loss paradox. One popular viewpoint is that information escapes from the black hole with Hawking radiation, even though this apparently violates causality. One might suspect that large causality violations are related to a huge Lorenz boost of the stationary observer at infinity relative to the stationary observer at the stretched horizon. The boost may "magnify" nonlocal effects inherent in string theory (see [32] and references therein). There are some concrete calculations in string field theory supporting this scenario [31,32]. If this scenario is correct, then stringy effects can be large even when all curvature invariants are small. Perhaps quasilocal field theories on a curved background could provide a useful effective description of such situations. It is certainly suggestive that in quasilocal theories the notion of an approximately local observable only makes sense relative to a particular reference frame.

\section{ACKNOWLEDGMENTS}

I would like to thank Ofer Aharony, Korkut Bardakci, and Nati Seiberg for useful comments. This work was supported by a DOE grant DE-FG02-90ER4054442.
[1] E. Witten, "Some comments on string dynamics," hep-th/9507121; Nucl. Phys. B463, 383 (1996).

[2] A. Strominger, Phys. Lett. B 383, 44 (1996).

[3] O.J. Ganor and A. Hanany, Nucl. Phys. B474, 122 (1996).

[4] N. Seiberg and E. Witten, Nucl. Phys. B471, 121 (1996).

[5] M.J. Duff, H. Lu, and C.N. Pope, Phys. Lett. B 378, 101 (1996).

[6] K. Intriligator, Nucl. Phys. B496, 177 (1997).

[7] J.D. Blum and K. Intriligator, Nucl. Phys. B506, 199 (1997).

[8] M. Berkooz, M. Rozali, and N. Seiberg, Phys. Lett. B 408, 105 (1997).

[9] N. Seiberg, Phys. Lett. B 408, 98 (1997).

[10] W. Nahm, Nucl. Phys. B135, 149 (1978).

[11] O. Aharony and T. Banks, J. High Energy Phys. 03, 016 (1999).
[12] S. Weinberg, The Quantum Theory of Fields. Vol. 1: Foundations (Cambridge University Press, Cambridge, England, 1995).

[13] V.Ya. Fainberg and M.A. Soloviev, Ann. Phys. (N.Y.) 113, 421 (1978).

[14] O. Aharony, M. Berkooz, D. Kutasov, and N. Seiberg, J. High Energy Phys. 10, 004 (1998).

[15] A.W. Peet and J. Polchinski, Phys. Rev. D 59, 065011 (1999).

[16] S. Minwalla and N. Seiberg, J. High Energy Phys. 06, 007 (1999).

[17] O. Aharony and M. Berkooz, J. High Energy Phys. 10, 030 (1999).

[18] A. Giveon, D. Kutasov, and O. Pelc, J. High Energy Phys. 10, 035 (1999).

[19] A. Giveon and D. Kutasov, J. High Energy Phys. 10, 034 (1999); 01, 023 (2000). 
[20] R.F. Streater and A.S. Wightman, PCT, Spin And Statistics, And All That (Addison-Wesley, Redwood City, CA, 1989).

[21] I.M. Gel'fand and G.E. Shilov, Generalized Functions (Academic, New York/London, 1968), Vol. 2.

[22] A. Jaffe, Phys. Rev. 158, 1454 (1967).

[23] N.N. Bogolyubov, A.A. Logunov, and I.T. Todorov, Introduction To Axiomatic Quantum Field Theory (Benjamin, Reading, MA, 1975).

[24] M.Z. Iofa and V.Ya. Fainberg, Zh. Éksp. Teor. Fiz. 56, 1644 (1969) [Sov. Phys. JETP 29, 880 (1969)].

[25] M.A. Soloviev, Theor. Math. Phys. 7, 183 (1971); 20, 299 (1974).
[26] V.Ya. Fainberg and M.A. Soloviev, Commun. Math. Phys. 57, 149 (1977)

[27] G.E. Shilov, Dokl. Akad. Nauk SSSR 102, 893 (1955).

[28] L. Hörmander, An Introduction to Complex Analysis in Several Variables (North-Holland, Amsterdam, 1990).

[29] M.Z. Iofa and V.Ya. Fainberg, Theor. Math. Phys. 1, 187 (1969).

[30] G.V. Efimov, Commun. Math. Phys. 5, 42 (1967).

[31] D.A. Lowe, L. Susskind, and J. Uglum, Phys. Lett. B 327, 226 (1994).

[32] D.A. Lowe, J. Polchinski, L. Susskind, L. Thorlacius, and J. Uglum, Phys. Rev. D 52, 6997 (1995). 